\title{
Angiographic diagnosis of anomalous coronary artery in tetralogy of Fallot
}

\author{
Julene S Carvalho, Celia M C Silva, Michael L Rigby, Elliot A Shinebourne
}

Department of

Paediatric Cardiology,

Royal Brompton

National Heart and

Lung Hospital,

London

J S Carvalho

M L Rigby

E A Shinebourne

Disciplina de

Cardiologia, Escola

Paulista de Medicina,

São Paulo, Brazil

C M C Silva

Correspondence to:

Dr E A Shinebourne,

Department of Paediatric

Cardiology, Royal

Brompton National Heart

and Lung Hospital, Sydney

and Lung Hospital, Sydney

Accepted for publication

21 January 1993

\begin{abstract}
Objective-To obtain angiographic views in tetralogy of Fallot that can show whether or not an anomalous coronary artery passes anterior to the right ventricular outflow tract.
\end{abstract}

Design-(a) A 10 year retrospective review of all patients who underwent repair of tetralogy of Fallot up to December 1990; (b) a prospective study of 30 children undergoing routine cardiac catheterisation.

Patients and methods-295 cases in whom standard angiographic views had been used were reviewed retrospectively. Thirty non-consecutive children with tetralogy of Fallot were studied prospectively, including one child previously studied in whom diagnosis of an unsuspected anomalous coronary artery was made only at operation. The aortogram was performed with $\geqslant 45^{\circ}$ caudocranial and $20^{\circ}-30^{\circ}$ left anterior oblique angles.

Setting-Tertiary referral centre.

Results-Ten of the 295 cases reviewed were shown to have a coronary vessel traversing the right ventricular outflow tract. In one case the diagnosis was suspected before operation but it was missed in the others. Even in retrospect we could not be certain of the precise anatomy with the use of standard angiographic views. In the prospective study the caudocranial aortogram showed the aortic valve face on in all the patients. The right ventricular outflow tract lay in a left and anterior (seen as superior) position in relation to the aortic root. Thus any vessel crossing the outflow tract could be identified. Identification of the aortic cusps allowed precise definition of the origin of the coronary arteries. All but four had normal origin and course of the coronary arteries. Four had paired left anterior descending arteries (including the restudied patient), in all cases with a large vessel originating from the right coronary artery passing across the right ventricular outflow tract.

Conclusions-Important anomalies of the coronary arteries in tetralogy of Fallot may remain undiagnosed if standard angiographic projections are used. Aortography with $\geqslant 45^{\circ}$ caudocranial and $20^{\circ}-30^{\circ}$ left anterior oblique angles allows precise definition of the anatomy and certainty as to whether any major vessel crosses the right ventricular outflow tract. Interpretation, however, can only be correct if the projection is technically adequate with a view of the aortic valve face on. Furthermore, a normal bifurcation of the left main stem does not exclude a second left anterior descending artery crossing the pulmonary outflow tract.

\section{(Br Heart f 1993;70:75-78)}

Tetralogy of Fallot is one of the conditions for which cardiac catheterisation is still performed before operation in most centres. This is because non-invasive techniques may not always permit identification of abnormalities of pulmonary arteries or of coronary artery anatomy. The sensitivity and specificity of angiography for diagnosing coronary anomalies, however, remains uncertain. Surgical and postmortem series suggest that important coronary anomalies occur in $2 \%$ to $10 \%$ of patients. ${ }^{1-3}$ The most common anomaly, the left anterior descending artery arising from the right coronary artery and crossing the right ventricular outflow tract, is important in young infants undergoing complete repair. It may influence surgical mortality and morbidity and has implications regarding technique and age of repair. Though age in itself may not represent an incremental risk factor for surgery, ${ }^{45}$ preoperative diagnosis of a vessel crossing the right ventricular outflow tract remains a contraindication for early repair if the use of an extracardiac conduit is to be avoided.

We have found it difficult to make the diagnosis with certainty based on standard angiographic views and decided to review our 10 year experience. The use of a caudocranial aortogram has been described previously in patients with aortic valve disease. ${ }^{6}$ More recently its use in children with transposition of the great arteries has been reported. ${ }^{7}$ This prompted us and others ${ }^{8}$ to assess its use in tetralogy of Fallot. A prospective study was then carried out with this purpose.

\section{Patients and methods}

To obtain retrospective data records of patients who underwent complete repair of tetralogy of Fallot at the Royal Brompton Hospital from January 1980 to December 1990 were reviewed.

To obtain prospective data we studied 30 non-consecutive patients with tetralogy of 
Fallot undergoing routine cardiac catheterisation. This included one child with an anomalous left anterior descending coronary artery missed at previous cardiac catheterisation but identified at surgery.

Twenty had a left and 10 a right sided aortic arch. Age varied from one month to 11.8 years (median 17 months). Either a retrograde $(n=25)$ or anterograde approach $(n=$ 5) was used for the biplane aortogram, which was performed with a minimal $45^{\circ}$ caudocranial and $20^{\circ}-30^{\circ}$ left anterior oblique angulation as well as a straight lateral projection. In four of the cases studied by a retrograde approach aortography was performed with a pigtail catheter but in general a standard National Institute of Health catheter gave better opacification of the coronary arteries. When possible, however, an anterograde approach with a balloon tipped catheter with the balloon inflated in the ascending aorta, was preferable.

\section{Results}

RETROSPECTIVE STUDY

Records for 295 patients were reviewed. Ten (five males and five females) had an associated anomalous coronary artery. Age at time of repair ranged from 10 months to $7 \cdot 6$ years. All had undergone preoperative echocardiography and cardiac catheterisation. Aortography and left ventriculography had been performed in one or two of the following views: anteroposterior, lateral, or left anterior oblique for the aortogram and lateral, long axis, or right oblique for the left ventriculogram. In only one case was there a high degree of doubt that an abnormality of coronary artery anatomy was present. Six had had previous palliative surgery. One of these had first stage correction (relief of the right ventricular outflow tract but no closure of the ventricular septal defect) and an anomalous vessel was identified at surgery. Subsequently, but before definitive repair, angiography was carried out with selective coronary artery injections. In this case, all six standard views, anteroposterior, lateral, right and left anterior oblique with and without craniocaudal tilt, were used but still there was no certainty as to the course of the vessel passing in front of the right ventricular outflow tract. In all cases it was necessary to modify the technique of repair as the usual incision to the right ventricular outflow tract could not be made. A combination of modified right ventriculotomy, right atriotomy, and pulmonary arteriotomy was used. Good relief of right sided obstruction was achieved in all patients but four required interposition of a conduit between the right ventricle and the pulmonary artery.

\section{PROSPECTIVE STUDY}

Selective coronary arteriography (fig 1) in one case and placement of a second catheter in the right ventricular outflow tract (fig 2) in another facilitated understanding and confirmed how the anatomy is displayed in the caudocranial angiographic projection with the left oblique angle. In a third case the aorta was reached by an anterograde route with the venous catheter and spillage of contrast into the right ventricle and the right ventricular outflow tract by accident contributed further to the understanding of the relation of the coronary arteries to the right ventricular outflow tract (fig 3). The course of the left anterior descending artery was seen by looking at the interventricular septum with the same angles during left ventriculography in two cases. Four patients (including the patient with a known abnormality) were shown to have a large left anterior descending artery from the right coronary artery as well as a second left descending artery from the left coronary cusp, so two vessels were supplying the area of the left anterior descending artery (figs 4 and 5). In the remaining 26 patients the coronary distribution was normal and none had an abnormal branch across the area
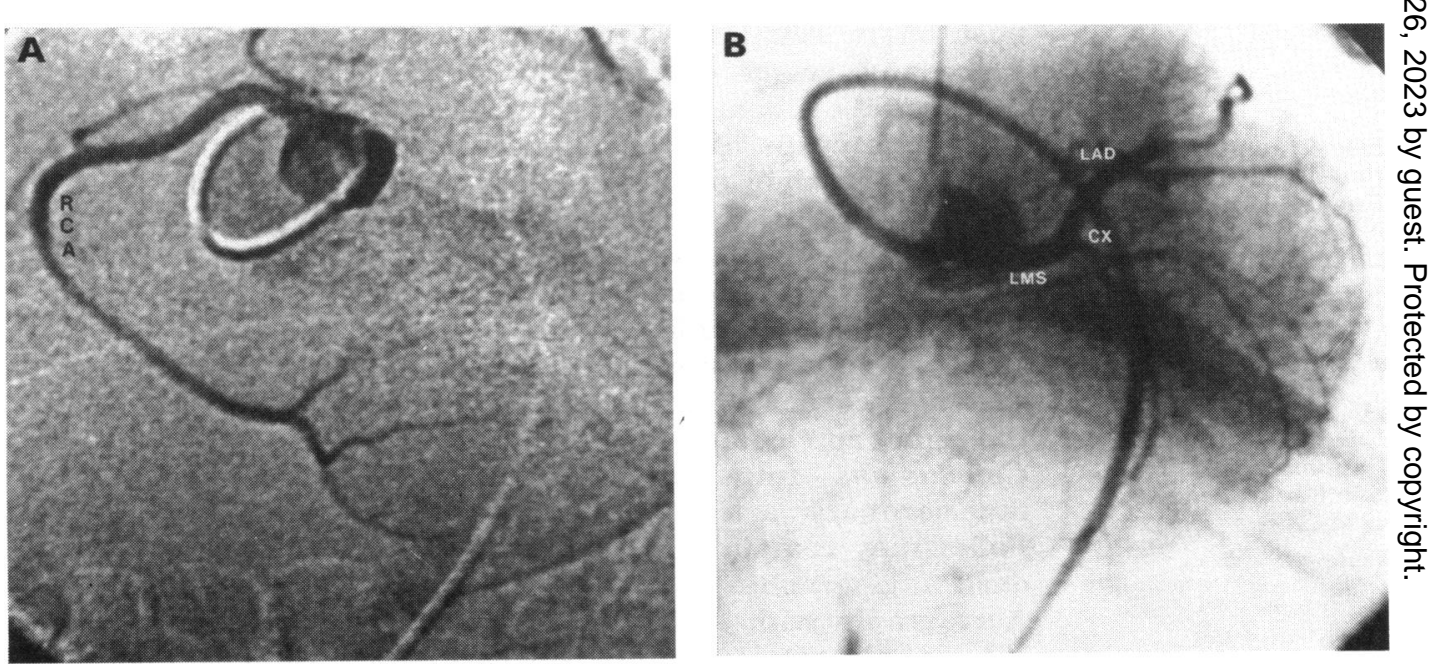

Figure 1 Selective right $(A)$ and left $(B)$ coronary artery injections displayed in the caudocranial left anterior oblique view in a patient with tetralogy of Fallot and normal distribution of coronary arteries. Note the course of the vessels as they arise from the right $(A)$ and left $(B)$ coronary sinuses. Cx, circumflex artery; $L A D$, left anterior descending artery; LMS, left main stem; $R C A$, right coronary artery. 


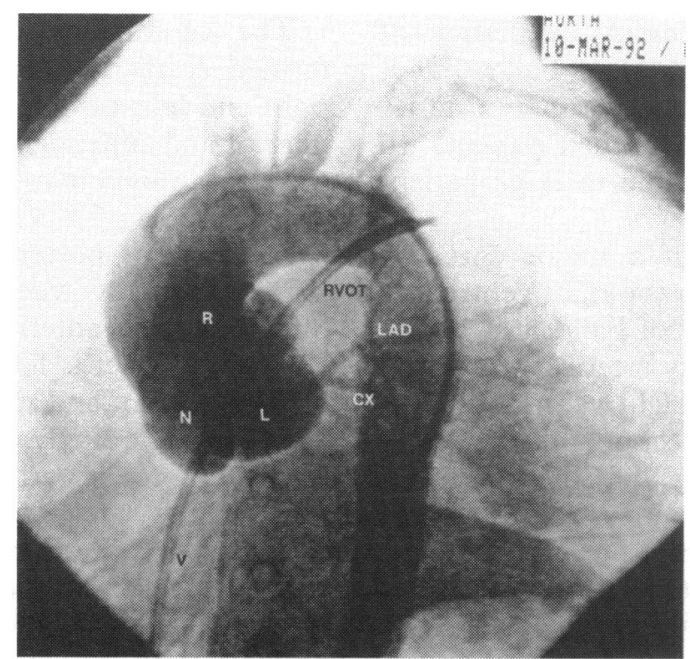

Figure 2 Retrograde arterial aortogram taken with a simultaneous venous catheter placed in the outflow tract of the right ventricle from a patient with tetralogy of Fallot. Note the three cusps of the aortic valve and the two branches of the lefi coronary artery. Cx, circumflex artery; $L A D$, left anterior descending artery; $L$, left coronary cusp $N$, non-coronary cusp; $R$, right coronary cusp; $R V O T$, right ventricular outflow tract; $V$, venous catheter.

of the right ventricular outflow tract. In three cases the conus branch, arising from the right coronary artery, was enlarged but did not run across the outflow tract area. In 21 cases the anatomy was known or was confirmed at time of surgery, including three of four with abnormality. In one, the vessel originating from the right coronary artery and supplying the left descending territory was considered smaller than the artery coming from the left coronary sinus. Relief of the right ventricular outflow tract obstruction was through the pulmonary artery and right atrium. Three patients have undergone palliative procedures, including one with abnormality. The rest await surgical treatment.

The lateral aortogram performed simultaneously was unhelpful in assessing the coronary artery anatomy with certainty but was

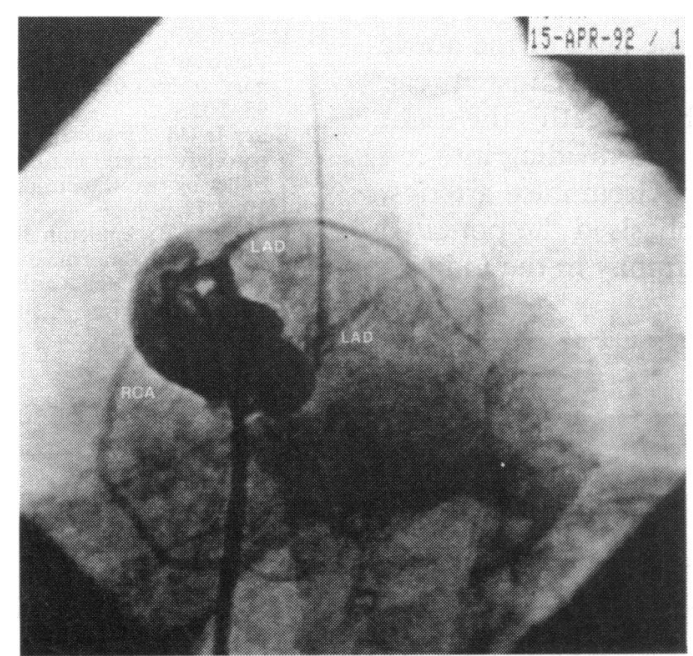

Figure 4 Anterograde venous aortogram from a patient with tetralogy of Fallot and anomalous coronary artery. Note that a large left anterior descending artery arises anomalously from the right coronary artery and traverses the area of the right ventricular outflow tract. A second, smaller descending artery is also seen. LAD, left anterior descending artery; $R C A$, right coronary artery.

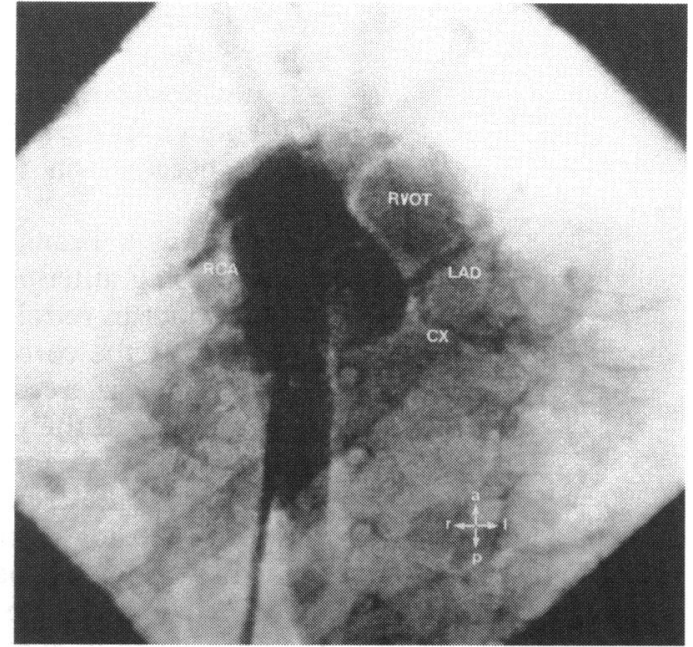

Figure 3 Anterograde venous aortogram from a patient with tetralogy of Fallot. Note the normal relations of the two coronary arteries to the right ventricular outflow tract $a$, anterior; Cx, circumflex artery; l, left; $L A D$, left anterior descending artery; $p$, posterior; $r$, right; $R C A$, right coronary artery; $R V O T$, right ventricular outflow tract.

used to show the presence or absence of an arterial duct.

\section{Discussion}

Successful display of coronary artery anatomy in tetralogy of Fallot, reported by means of a ventriculogram or flush aortogram ${ }^{10}$ or with selective coronary artery injections has been reported. ${ }^{11}$ This has not been our experience with aortography and we do not carry out selective coronary arteriography routinely. Cross sectional echocardiography may also be used. ${ }^{12}{ }^{13}$ Although it is possible to identify abnormal vessels across the right ventricular outflow tract, there may not be an adequate view of the coronary anatomy in a significant proportion of patients. Berry et al correctly and blindly distinguished normal and abnor-

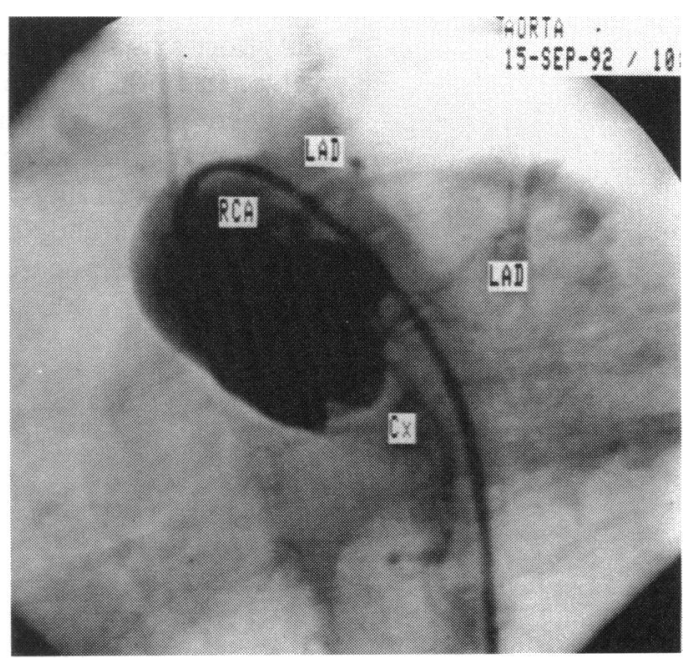

Figure 5 Retrograde arterial aortogram from a patient with tetralogy of Fallot. Note paired left anterior descending arteries. One passes across the pulmonary outflow tract, the other arises normally from the lefi main stem. Cx, circumflex artery; $R C A$, right coronary artery; $L A D$, left anterior descending artery. 
mal patterns in the coronary circulation of some patients with tetralogy of Fallot but it was not possible to see the coronary arteries well enough in over $20 \%$ of their patients. In only two cases was this due to large patient size. ${ }^{12}$

To show a branch of a major coronary artery passing anterior to the right ventricular outflow tract in tetralogy of Fallot it is necessary to show the coronary arteries clearly and also to know where the right ventricular outflow tract lies in the projection chosen for the aortogram. Overlapping of the coronary branches is the rule with most standard views. This leads to confusion and failure to recognise abnormal patterns when interpreting the angiograms. In our retrospective series the prevalence of surgically important anomalies of the coronary artery was $3.4 \%$ but the diagnosis was not made with certainty in any of the cases although suspected in one. Conversely, in our prospective series of patients in whom a caudocranial aortogram was performed, the diagnosis was made with certainty in four cases and, equally important, we could be certain that no major coronary artery passed anterior to the right ventricular outflow tract in the rest.

With the use of a caudocranial angle for the aortogram the aortic valve is seen face on. Its three cusps are clearly seen and the origin and course of the coronary arteries are easily followed by the absence of overlapping as pointed out by Mandel et al. ${ }^{7}$ Furthermore, and of crucial importance in patients with this condition, knowing that the position of the right ventricular outflow tract is to the left and slightly anterior to the aortic valve allows identification of any abnormal branch crossing the area. To be certain, however, a correct projection of the base of the heart is required (as if the observer were looking at the heart from below and slightly from the left). The aortic arch itself is foreshortened and therefore the aortic valve must be seen face on or nearly so. This view is thus analagous to the echocardiographic parasternal short axis section at the level of the aortic valve. In both, the normal left mainstem can be seen passing behind (beneath) the right ventricular outflow tract and dividing into left anterior descending and circumflex arteries, but we have already emphasised the potential deficiency of echocardiography in the individual patient.
The caudocranial aortogram, when performed correctly, allows distinction between right and left, posterior and anterior (but not inferior and superior) relations (fig 3). Thus it should be possible to detect an abnormal origin or course of a coronary artery and to'see its anterior or posterior relation to the pulmonary outflow tract. Any major coronary branch passing anterior to the right ventricular outflow tract is likely to be of importance to the surgeon, and management can then be directed accordingly. It is also important to see both coronary arteries as identification of one normal left anterior descending artery does not exclude a paired artery originating from the right coronary artery and crossing the pulmonary outflow tract. Our current practice is to use $45^{\circ}$ caudocranial and $30^{\circ}$ left anterior angulation in all patients. We have shown that by using this angiographic projection it is possible to detect these abnormalities before operation.

1 Berry BE, McGoon DC. Total correction for tetralogy of Fallot with anomalous coronary artery. Surgery 1973; 74:894-7.

2 Meng CC, Eckner FA, Lev M. Coronary artery distribution in tetralogy of Fallot. Arch Surg 1965;90:363-6.

3 Howe A, Rastelli GC, Ritter DG, Dushane JW, McGoon DC. Management of right ventricular outflow tract in severe tetralogy of Fallot. $\mathcal{f}$ Thorac Cardiovasc Surg severe tetralogy

4 Touati GD, Vouhe PR, Amodeo A, Pouard P, Mauriat P, Leca F, et al. Primary repair of tetralogy of Fallot in infancy. $\mathcal{F}$ Thorac Cardiovasc Surg 1990;99:396-403.

5 DiDonato RM, Jonas RA, Lang P, Rome JJ, Mayer JE, Castaneda AR. Neonatal repair of tetralogy of Fallot with and without pulmonary atresia. $\mathcal{F}$ Thorac Cardiovasc Surg 1991;101:126-37.

6 Stein PD, Sabbah HN. Orifice-view roentenography for evaluation of the aortic valve. American fournal of Roentgenology Radium Therapy and Nuclear Medicine 1975;125:847-53.

7 Mandell VS, Lock JE, Mayer JE, Parness IA, Kulik TJ. The 'Laid-back' aortogram: An improved angiographic The 'Laid-back' aortogram: An improved angiographic view for demonstration of coronary arteries in transposi-
tion of the great arteries. Am $f$ Cardiol 1990;65: tion of

8 O'Sullivan JJ, Bain HH, Hunter S, Wren C. The 'end-on' aortogram in tetralogy of Fallot: Improved angiographic definition of coronary arteries without selective angiography. Pediatr Cardiol 1992;13:247.

9 Fellows KE, Freed MD, Keane JF, Van Praagh $R$ Bernhard WF, Castaneda AC. Results of preoperative coronary angiography in tetralogy of Fallot. Circulation 1975;51:561-6.

10 Fellows KE, Smith J, Keane JF. Preoperative angiocardiography in infants with tetrad of Fallot. $\mathrm{Am} \mathcal{F}$ Cardiol 1981;47:1279-85.

11 Dabizzi RP, Caprioli G, Aiazzi L, Castelli C, Baldrighi G, Parenzan $L$, et al. Distribution and anomalies of coroParenzan $L, e t$ al. Distribution and anomalies of coronary arterie.

12 Berry Jr JM, Einzig S, Krabill KA, Bass JL. Evaluation of coronary artery anatomy in patients with tetralogy of Fallot by two-dimensional echocardiography. Circulation 1988;78:149-56.

13 Jureidini SB, Appleton RS, Nouri S. Detection of coronary artery abnormalities in tetralogy of Fallot by twodimensional echocardiography. $f^{\mathrm{Am}}$ Coll Cardiol 1989;14:960-7. 